\title{
An Investigation into the Use of Tactile Instructions in Snowboarding
}

\author{
Daniel Spelmezan \\ INRIA; Univ Paris-Sud \& CNRS (LRI) \\ F-91405 Orsay, France \\ spelmezan@lri.fr
}

\begin{abstract}
In many sports, athletes are spatially separated from their coach while practicing an exercise. This spatial separation makes learning new skills arduous because the coach cannot give instructions or feedback on performance. We present the findings of an in the wild study that demonstrate the potential for teaching sport skills with realtime tactile instructions. We focused on snowboard training. Ten amateurs learned a riding technique with a wearable system that automatically provided tactile instructions during descents. These instructions were in sync with the movements of the snowboard and signaled how to move the body. We found that tactile instructions could help snowboarders to improve their skills. We report insights into the snowboarders' opinion and give recommendations for teaching sport skills with tactile instructions. Our findings help to identify the conditions under which tactile instructions can support athletes in sports training.
\end{abstract}

\section{Author Keywords}

Tactile instructions; vibrotactile feedback; sports training; motor skill learning; snowboarding; wearable computing

\section{ACM Classification Keywords}

H.5.2 Information Interfaces and Presentation: User Interfaces_Evaluation/methodology, Haptic I/O

\section{INTRODUCTION}

For acquiring motor skills, such as in sports training, it is important that the learner frequently receives instructions on how to perform the skill and feedback on the performance. Coaches typically give instructions and feedback before and after a trial, and concurrently during the execution of the movements (Figure 1, left). Yet in many sports the coach cannot correct the learner during an exercise. A good case in point is snowboarding. Snowboarders receive instructions before descending the slope and delayed feedback after the ride. During the ride, they are spatially separated from their coach. They have to rely on their own perception of what is right or wrong (Figure 1, right).

\footnotetext{
D. Spelmezan. An Investigation into the Use of Tactile Instructions in Snowboarding. In MobileHCI '12: Proceedings of the 14th International Conference on Human Computer Interaction with Mobile Devices and Services, 417-426, ACM, 2012.

http://doi.acm.org/10.1145/2371574.2371639

(c) ACM, 2012. This is the author's version of the work. It is posted here by permission of ACM for your personal use. Not for redistribution. The definitive version will be published in MobileHCI'12, September 21-24, 2012, San Francisco, California, USA.
}
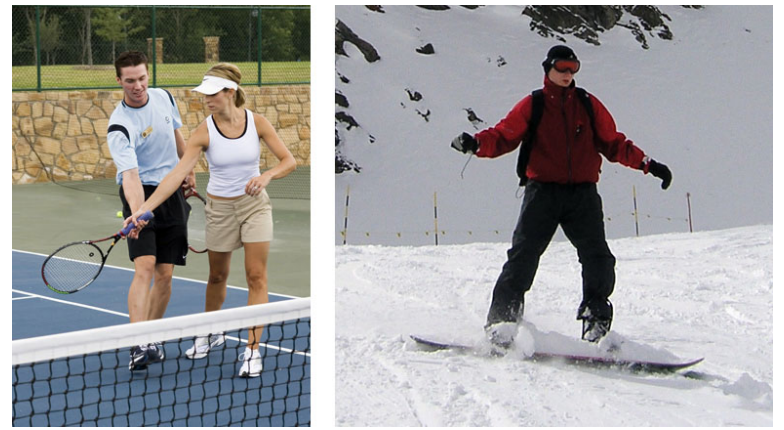

Figure 1. Left: The tennis student receives instructions and feedback while practicing an exercise. The coach can also guide her movements to demonstrate the correct technique. Right: The snowboard student is spatially separated from his coach during the ride. He typically does not receive instructions nor feedback while descending the slope. (Image on the left courtesy of Cooper Aerobics Center.)

For instructing snowboarders during the ride, the coach could descend alongside the student to call out instructions. Even so, snowboarders could miss these spoken messages in the noisy environment. Spoken messages could also block environmental audio cues on which snowboarders rely on.

Artificial tactile stimuli are an alternative means to give realtime instructions or feedback without these drawbacks. These stimuli can be generated by actuators, such as vibration motors that are sewn into the sportswear. Tactile stimuli have been proposed as application in sports for instructing athletes where to move to, how to move, and when to move [20]. Their advantage is that they directly stimulate the body, like a coach who guides the student's movements (Figure 1, left).

The findings of several studies indicate that artificial tactile stimuli could be beneficial for learning motor skills, such as dancing [14], rowing [20], and karate moves [3]. There is also evidence that tactile stimuli can facilitate the learning of musical instruments [9, 10, 21]. Even so, this previous work [14, 20, 3] did not consider realistic training scenarios but focused on laboratory settings, nor did it focus on sports training $[9,10,21]$. To better understand the potential and pitfalls of using tactile stimuli for teaching sport skills, it is important to consider a broad range of physical activities, user groups, learning conditions, and real-world scenarios.

We are specifically interested in instructing snowboarders during the ride. Snowboarders experience physical strain and tactile sensations that occur naturally while moving their body. These natural sensations could interfere with artificial 
sensations that actuators evoke on the skin. Snowboarders also focus their attention on the sport, on their body movements, and on the environment. Thus, they can pay less attention to messages that they receive over the tactile sense. Our challenge is to find tactile stimuli that snowboarders can perceive and attend to in active situations. These instructions should not increase the snowboarders' cognitive load. Moreover, we have to determine the teaching and learning conditions under which realtime tactile instructions are effective.

Our work addresses several questions: Can snowboarders perceive tactile instructions when they practice a new riding technique in a realistic scenario? Can they attend to these instructions? How helpful do they consider these instructions? Under which learning conditions do tactile instructions support snowboarders in acquiring new skills, and which conditions should be avoided during training?

To find answers to these questions, we conducted a field study with ten snowboarders who participated in a snowboarding course for one day. These snowboarders learned an unfamiliar riding technique with traditional lessons where the coach provided spoken instructions and feedback before and after the ride, and with lessons where they additionally received tactile instructions while descending the slope. A custom-built wearable system automatically instructed the snowboarders during the ride. This system sensed the movements of the snowboard and provided tactile instructions at the point in time when the snowboarders had to perform body movements that were essential to their riding technique.

This work investigates the use of realtime tactile instructions for snowboard training in a realistic scenario. We first discuss related work and our research goals. We then describe our wearable snowboard training system and the coach's opinion, who tested the system on the slope. After describing the snowboarding course, we discuss our findings on the effectiveness of tactile instructions and the snowboarders' opinion. We conclude with recommendations for applying tactile instructions during training.

\section{RELATED WORK}

Sport technologies use embedded and wearable sensors for measuring physiological aspects and muscle movements that can help coaches to understand and to improve the performance of athletes. Chi et al. [4] gave an introduction to this topic and described that these technologies could help to improve sports performance and learning, to encourage more exercises, and to make sports more entertaining.

Besides assisting coaches, sport technologies could also support athletes with realtime instructions on how to move the body or with immediate feedback on their performance. This information could be presented as artificial tactile stimuli, as sound, or visually on a display.

\section{Tactile Instructions}

When the athlete needs only a hint at how to adjust the posture, a tactile stimulus can nudge the body in the right direction. Van Erp et al. [20] described that tactile stimuli could signal to athletes where to move to, how to move, and when to move. Van Erp et al. [20] also reported that the first two application scenarios-where and how to move-were tested with elite athletes in soccer training, cycling, and speed skating. Furthermore, van Erp et al. [20] conducted a laboratory study where rowers received tactile instructions when to move the legs and the back while exercising with a rowing machine. The findings of their study indicated that tactile instructions can help athletes to maintain a high performance level.

Several studies suggest that tactile instructions can support the learner in acquiring motor skills. Bloomfield et al. [3] reported that tactile stimuli at the arm help novices to learn karate arm movements in a virtual reality setup. Nakamura et al. [14] applied tactile stimuli at the wrist to instruct dance beginners when to perform a movement. In general, the participants were able to increase the number of correct movements and to perform these movements faster compared to dancing without tactile cues. Tactile stimuli were also shown to be effective for learning to play musical instruments. For example, Holland et al. [9] used tactile stimuli at the wrists and ankles for teaching drum patterns. Huang et al. [10] used tactile stimuli at the fingers for teaching piano melodies.

In our previous work [16], we focused on designing tactile stimuli that could intuitively represent body movements. We also reported that snowboarders were able to perceive and to identify tactile instructions while descending a slope. We did not, however, consider a realistic training scenario.

\section{Tactile Feedback}

Besides instructing when and how to perform a movement, a tactile stimulus can also act as feedback when the movement is wrong. In general, feedback does not instruct the learner how to perform a movement but it can implicitly indicate the direction to move in order to correct the error. For example, Lieberman et al. [13] asked participants to mimic an arm movement that was shown on a computer display. A motion capture system tracked and analyzed the performed movements. Realtime tactile feedback at the arm represented the deviations from the target movements. This feedback resembled a force-field around the correct movement path and indicated the intended movement direction. According to the findings of their laboratory study, Lieberman et al. reported that the addition of tactile feedback to motor training could improve performance and could support learning.

Van der Linden et al. [21] conducted a two-months study where tactile feedback supported children in playing the violin in a realistic teaching scenario. Similar to [13], they used a motion capture suit for tracking the children's movements and posture while bowing. They found that realtime tactile feedback at the arms and torso enabled the children to improve the holding of the violin and straight bowing. Moreover, both the teachers and the children used the system in new ways to achieve their own objectives.

Finally, haptic devices that generate force feedback have been used for motor skill training in virtual environments. For example, Feygin et al. [6] reported that haptic guidance helped users to learn the temporal aspects of the motor skill, whereas visual training was better for learning the motion shape. 


\section{Audio and Visual Feedback}

For elite sports training, Baca et al. [1] explored rapid feedback systems for rowing, table tennis, and shooting. These systems sensed relevant performance data and visualized this data as graphs on a computer display shortly after motion execution. For martial arts training, Kwon et al. [12] combined data from accelerometers with video analysis for visualizing the power of punches as circles of different sizes. Takahata et al. [18] used accelerometers for analyzing the timing and the forces of wrist, waist, and ankle movements. These measurements were mapped to sound patterns that enabled novices to hear and to compare the characteristics of their movements to the instructor's movements while punching.

A similar training tool is the Sonic Golf Club (see sonicgolf.com). This club measures the speed of the swing with built-in accelerometers, and maps the speed to a sound pattern that is played back over earplugs. The interactive throwing sleeve is a high tech-armband with sensors at the wrist and elbow [19]. Intended for sports such as basketball, this tool measures the athlete's arm movements. A remote computer analyzes the data and maps the result to musical cues. Both tools provide instant feedback that enables athletes to hear the characteristics of their movements and to recognize movements that lead to successful shots.

Realtime sound feedback on performance has also been applied to skiing. A common mistake in skiing is to tip the shoulders during turns. The Ski Coach (see theskicoach.com) is a mechanical device that generates an audible clink when the skier performs turns without tipping the shoulders.

Although these training tools give athletes immediate feedback on their performance, audio and visual feedback can have disadvantages. Sound can be difficult to perceive in noisy environments and can also block environmental audio cues. Computer displays typically restrict training to laboratory settings and require athletes to divide their attention between the sport and interpreting the displayed data.

\section{RESEARCH GOALS}

The aforementioned findings on using tactile instructions and tactile feedback for motor skill learning suggest that artificial tactile stimuli can assist learners in acquiring motor skills. These findings, however, cannot be generalized to sports training because the application domain and the movement tasks are different. Moreover, only a few studies were conducted under real-world conditions. Van der Linden et al. [21] stressed the importance of conducting studies in the wild, as the experiences of a lesson under laboratory conditions differ from the experiences in realistic scenarios.

An important aspect to consider is that physical exertion and cognitive load can degrade the learner's ability to perceive and to attend to tactile stimuli. For example, Bhargava et al. [2] reported that young adults who floated freely during zero-gravity flights in an airplane were not able to reliably identify the location of tactile stimuli on their body. Van der Linden et al. [21] reported that children did not notice tactile feedback in situations where they experienced high cognitive load. Also, Hoggan et al. [8] reported that tactile feedback

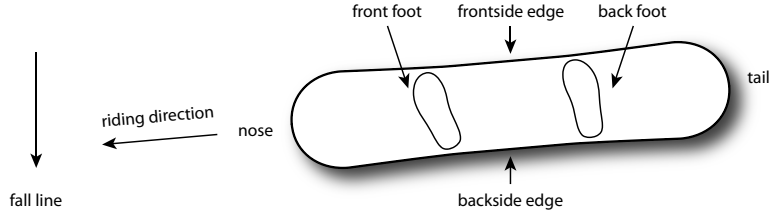

Figure 2. Snowboard terminology and typical stance when the left foot points forward. Alternatively, some snowboarders prefer to descend with the right foot pointing forward. The fall line is the most direct path that a ball would roll down the hill.

for a touchscreen typing task becomes ineffective in an environment with high background vibration levels. Although the findings of several studies indicate that the participants were able to perceive and to respond to tactile stimuli under $\operatorname{cog}$ nitive load and in active situations [9,21, 16, 20], it is not known under which conditions tactile stimuli will become ineffective as instructions in sports training.

Finally, it is not known what athletes and coaches think about teaching sport skills with tactile instructions. To give first insights into these issues, we focused on a realistic training scenario. We chose snowboarding as example activity because this sport requires athletes to continuously adjust their movements and posture, instead of performing repetitive movements with a fixed movement range. Snowboarders also perform open skills [22], which involves body movements that they have to execute under time-pressure, without planning them, and in an environment that can change during the execution of the movements. We expected to identify conditions under which tactile instructions should be applied during training, and to gain insights into their use that could also benefit other sports and physical activities.

\section{THE SNOWBOARD TRAINING SYSTEM}

To illustrate the aspects of snowboarding that we addressed with tactile instructions, we will briefly introduce the basic technique for descending the slope and two typical riding mistakes. We will then describe the wearable system and the tactile instructions that we used in our study.

\section{Fundamentals of Snowboarding}

A snowboard has two edges and resembles a wide ski. Both feet are fixed to the snowboard such that the stance is transverse to the direction of travel (Figure 2). During the ride, the weight is distributed evenly between the feet. The shoulders and hips are in line with the feet's stance. Snowboard coaches refer to this posture as neutral position [5].

Descending the slope involves a sequence of turns that alternate between riding on the frontside edge and riding on the backside edge. To switch from one edge to the other edge, the rider points the snowboard downhill and pivots across the fall line. The basic technique for pivoting the snowboard is called basic turn. This turn involves a sequence of body movements. Assuming that the left foot points forward during the descent (Figure 2), the rider shifts the weight to the front foot and rotates the upper body to the left, towards the new riding direction. The resulting posture leads the snowboard to follow these movements, to align to the fall line, and to pivot to the backside edge. After pivoting the snowboard, 


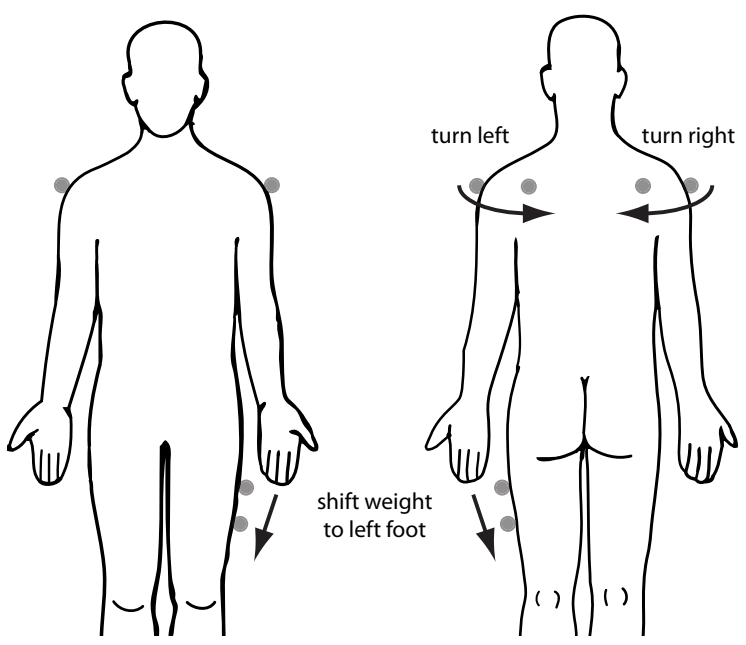

Figure 3. Two vibration motors were placed around each shoulder and laterally at the thigh that pointed forward during the ride. The arrows illustrate the direction of the stimuli on the skin. The labels describe the messages that the stimuli represented.

the rider adopts a neutral position: he redistributes the weight evenly between the feet and aligns the upper body parallel to the snowboard. For the next turn, in order to pivot the snowboard to the frontside edge, the rider shifts the weight to the front foot and rotates the upper body to the right.

This movement sequence challenges snowboard beginners and can lead to two typical mistakes: incorrect weight distribution and counter-rotation [5]. Facing downhill, many snowboarders tend to shift their weight to the back foot. This posture feels safer but makes it difficult to pivot the snowboard and can lead to falls. Also, they do not properly align the upper body towards the new riding direction. Instead, they counter-rotate the torso in the opposite direction.

\section{Tactile Instructions for Basic Turns}

We used two tactile instructions to address these mistakes. These instructions signaled which body movements to perform in order to pivot the snowboard with a basic turn. The first instruction stimulated the thigh and prompted the rider to shift the weight to the front foot. The second instruction stimulated either the left shoulder or the right shoulder, which depended on the direction of the new turn (Figure 3).

We based these instructions on the findings of our previous study [16], which focused on the intuitive interpretation of tactile stimuli. We reported that users preferred directional lines as instructions because they could often associate these lines with body movements. Therefore, we used the sensory saltation phenomenon [7] to draw directional lines on the skin [17]. The motors were sequentially activated. Each motor was pulsed three times for $100 \mathrm{~ms}$ with pauses of $50 \mathrm{~ms}$.

For motor skill learning, instructions on how to move the body should guide the learner's attention to the effects of body movements (external focus) instead of to the body (internal focus) [22]. For this reason, we worded the meaning of the tactile instructions to promote an external focus of attention, as noted in [16]. This wording was based on the spoken instructions that coaches use during training. The stimuli at the thigh, which signaled to shift the weight to the front foot, represented the message Increase the pressure towards the nose of the snowboard. The stimuli at the shoulder, which signaled to turn left or right, represented the message Hello mountain or Hello valley. This message depended on the riding direction after pivoting across the fall line, when the rider faced either the mountain or the valley.

\section{Hardware Setup for Automatic Tactile Instructions}

A custom-built wearable system automatically triggered the two tactile instructions during the ride. This system was based on an Arduino BT (see arduino.cc) and a Nokia N70 mobile phone as host device. We programmed the system to detect the point in time when the rider pivoted the snowboard from one edge to the other edge, as described in [15].

To sense the riding edge, we used four pressure sensors that measured the rider's weight distribution on the snowboard. One sensor was placed under each heel. Another sensor was placed under the toes. The difference between the pressure that was measured under the toes and under the heels yielded the riding edge. When the weight was towards the toes, the rider descended on the frontside edge (Figure 2). When the weight was towards the heels, the rider descended on the backside edge. Transitions between the frontside edge and the backside edge corresponded to pivoting the snowboard.

This sensing technique could not detect whether the snowboarder's posture was correct or wrong. For this reason, the system automatically provided the two tactile instructions whenever the rider pivoted the snowboard. Thus, the snowboarder received instructions for every turn.

We used off-the-shelf cylindrical vibration motors for mobile phones as actuators (type Nokia 3270). These motors vibrated at a frequency around $180 \mathrm{~Hz}$ when powered by four AA batteries. The motors were fixated under a tight T-shirt that had small pouches for inserting actuators.

\section{PILOT TEST AND THE COACH'S OPINION}

A snowboard instructor from RWTH Aachen University's sports center volunteered to conduct a snowboarding course for our study. Before the course took place, he tested the wearable system on the slope. To experience when the system triggered tactile instructions and what the evoked sensations felt like, he varied his speed and riding techniques, including basic turns, wide and short turns, riding switch, and carving. He confirmed that the system functioned as intended and that he received instructions whenever he started a new turn.

He suggested, however, to use shorter tactile stimuli because the compound instructions were too long (1750 ms). During short turns at high speed, the two instructions could interfere with body movements that the rider has to perform in order to return to neutral position after pivoting the snowboard. $\mathrm{He}$ also noticed that the onset of the instructions was sometimes slightly delayed when pivoting to the backside edge. Ideally, the instructions should occur at the very moment when the rider has to perform the body movements. This is while initiating a new turn before pivoting the snowboard, and while 


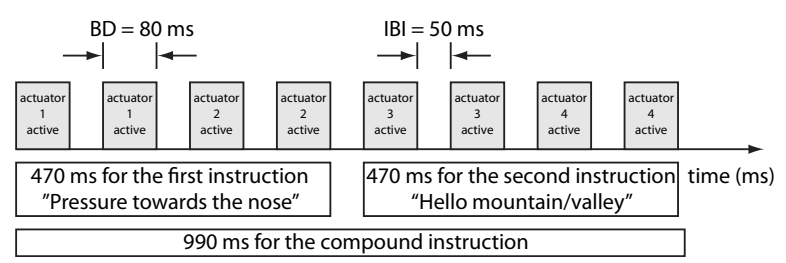

Figure 4. The tactile instructions were based on stimuli with a burst duration $\mathrm{BD}=\mathbf{8 0} \mathrm{ms}$ and an inter-burst interval IBI $=\mathbf{5 0} \mathrm{ms}$.

pivoting across the fall line. Even so, our coach considered this delay to be less critical with shorter tactile stimuli.

To shorten the length of the instructions, we experimented with shorter timing values and agreed to pulse each motor twice for $80 \mathrm{~ms}$. These values reduced the length of the compound instruction from $1750 \mathrm{~ms}$ to $990 \mathrm{~ms}$ (Figure 4).

\section{THE SNOWBOARDING COURSE}

Our goal was to explore if tactile instructions can support snowboarders in improving their riding skills. We were also interested in the snowboarders' opinion on receiving tactile instructions during the ride.

\section{The Task}

We decided to teach how to ride basic turns switch. This technique required the snowboarders to descend in an unfamiliar posture: the foot that normally pointed backward on the snowboard pointed forward. This entailed that snowboarders who preferred to descend with the left (right) foot pointing forward had to descend with the right (left) foot pointing forward (Figure 2). Riding switch makes snowboarders feel clumsy like beginners and is difficult to master even if they can descend the slope in their preferred stance.

\section{The Participants}

Ten university students volunteered (aged 21-29 years, two women). They were snowboarders at advanced beginner level who had basic snowboarding skills but who needed more focused sessions to refine their technique. On a scale ranging from level one (beginner) to level five (expert), three participants rated their snowboarding skills as level one, four as level two (advanced beginner), and three as level three (advanced). Six snowboarders had previously tried to ride switch. One participant was a first-time snowboarder. Before the course started, she received an one-hour introductory lesson to practice the basics of snowboarding.

\section{The Lessons}

The study took place in an indoor ski resort. We repeated the snowboarding course on five days with two participants per day. Donning and calibrating the wearable system lasted one hour. The lessons started in the morning and ended in the afternoon. A break of 45 minutes was scheduled at noon.

To allow all participants to experience lessons without tactile instructions and lessons with tactile instructions, we chose a within-subjects design with two conditions:

1. Traditional lessons where the coach provided spoken instructions before and spoken feedback after the descent.
2. Traditional lessons that were accompanied by tactile instructions when the rider pivoted the snowboard.

The order of the conditions was counterbalanced. In the morning, the experimenter randomly chose one participant $(P 1)$ to descend without tactile instructions (condition 1). The other participant $(P 2)$ descended with tactile instructions (condition 2). The conditions were reversed after the break. $P 1$ received tactile instructions (condition 2), whereas $P 2$ did not receive tactile instructions (condition 1).

We conducted a blind study to prevent bias on the coach's part. The coach did not know if his students received tactile instructions during the ride. This setup ensured that he treated all students alike when explaining exercises and when providing feedback on their performance after the ride. The participants carried the wearable system in a pouch and were asked not to reveal if they received tactile instructions.

The slope was $520 \mathrm{~m}$ (1700 ft) long. All participants descended ten times in this order:

- One descent for reference

- Four descents for one condition

- Four descents for the other condition

- One descent for reference

The coach used the first descent in the morning and the final descent in the afternoon for rating the participants' skills in riding switch before the course and after the course. The participants did not receive spoken instructions before nor spoken feedback after these reference trials. Neither did they receive tactile instructions while descending the slope.

The course started after the initial descent. The coach explained that tactile instructions during the ride indicate the body movements to perform in order to pivot the snowboard The experimenter manually triggered the instructions to allow the participants to learn the meaning of the stimuli. This procedure was repeated after the break.

For each of the four descents in the morning and in the afternoon, the coach issued an exercise that his students had to practice. He explained and demonstrated the required movements on level ground at the top of the slope. Then, he slowly descended the first half of the slope to demonstrate this exercise. From the middle of the slope, he signaled the first candidate to descend up to the location where he was waiting. After informing this participant how to improve the riding technique, he observed and advised the second participant Lastly, the group descended the remaining half of the slope. This time the coach did not correct his students after the ride, which was similar to real courses where the coach cannot give feedback after every run. Even so, the participant who was chosen to descend with tactile instructions did receive these instructions until the end of the slope.

The coach issued exercises as he considered appropriate for teaching basic turns. All participants practiced the same exercises in the morning. Some of these exercises, however, were less appropriate for the skilled snowboarders who quickly 


\begin{tabular}{|lccccccc|}
\hline Participant & C & D & E & F & G & H & J \\
\hline Recognized turns (\%) & 100 & 87.0 & 100 & 100 & 51.6 & 100 & 100 \\
\hline
\end{tabular}

Table 1. Percentage of turns where the system provided instructions while pivoting (incomplete sensor recordings for participants $A, B, I$ ).

progressed during the course. Therefore, the coach also issued more demanding exercises in the afternoon. He asked the advanced snowboarders to increase the edging angle of the snowboard and to carve the turns. Carving requires the rider to descend at higher speed, to adopt a posture that resembles the neutral position, and to pivot the snowboard before passing the fall line. As we did not design the tactile instructions for carving, they were reinterpreted to indicate possible riding mistakes. In this case, the coach asked his students to regard the tactile stimuli as a reminder for avoiding incorrect weight distribution and incorrect upper body rotation because these mistakes could also occur in carving.

\section{Data Acquisition and Analysis}

We recorded the course on footage in order to assess the snowboarders' performance off-line. For videoing the participants, the experimenter used a location at the edge of the slope, 230 meters away from the top. This location was above the coach's observation point, who observed his students from the middle of the slope. To evaluate the footage, we recruited another snowboard instructor who did not know about the objective of our study. In addition, the wearable system logged the sensor recordings. We evaluated these recordings off-line in order to assess how accurately the system recognized the pivoting process. This analysis allowed us to identify potential problems with the technology that could have influenced the participants' opinion on tactile instructions. Finally, we interviewed the participants after the course in order to gain insights into their learning experience.

\section{FINDINGS AND DISCUSSION}

\section{The Snowboard Training System}

The proper functioning of the wearable system was essential for instructing the snowboarders during the ride. We analyzed the sensor recordings and the footage in order to evaluate how accurately the system detected the point in time when the participants pivoted the snowboard.

The footage revealed that the participants performed on average 7.2 turns $(S D=1.54, \min =5, \max =11)$ when descending the first half of the slope with tactile instructions. The system recognized the pivoting of the snowboard on average in $90.7 \%$ of these turns. The system worked less accurately for the participants $D$ and $G$ (Table 1). We surmise that the recognition accuracy could improve with additional sensors and with a different sensor setup. For example, sensors could be integrated into the edges of the snowboard or inside the soles of the boots in order to avoid sensor displacement. This setup could also speed-up donning and simplify calibration.

To estimate the period when the system triggered tactile instructions, we marked on footage the turning points on the participants' riding paths (Figure 5 (a)). Based on the timestamps of the footage and of the sensor recordings, we calculated the offset between the time when the snowboarders

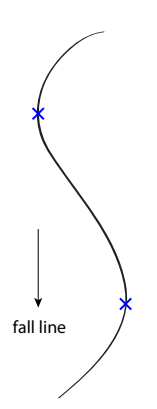

(a)

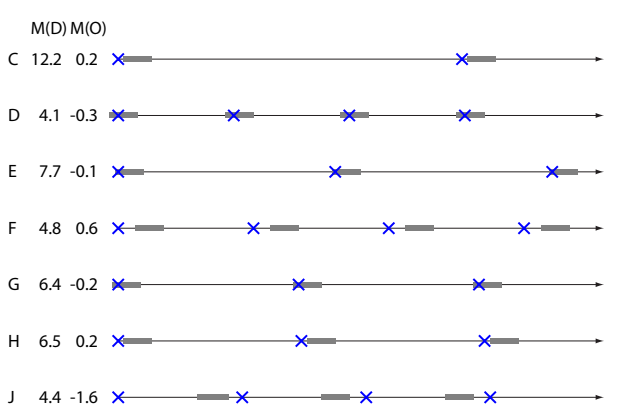

(b)
Figure 5. (a) The crosses indicate the turning points on the riding path when the snowboarders passed the fall line. (b) The rectangles show when they perceived the compound instructions $(990 \mathrm{~ms}$ long $)(\mathrm{M}(\mathrm{D})=$ average duration of turns, $M(O)=$ average time offset between passing the fall line and the onset of tactile instructions (in second)).

\begin{tabular}{|lcccccccccc|}
\hline Participant & A & B & C & D & E & F & G & H & I & J \\
\hline \hline Previous riding experience & y & y & n & y & y & y & n & n & n & y \\
\hline Rating before the course & 4 & 7 & 2 & 6 & 2 & 2 & 3 & 2 & 2 & 5 \\
Rating after the course & 6 & 8 & 4 & 7 & 4 & 5 & 6 & 4 & 5 & 7 \\
\hline
\end{tabular}

Table 2. The participants' previous experience in riding switch (yes/no). The coach's rating of their skills $(1=$ very bad, $10=$ very good; participants with initially poor riding skills are shown in bold font).

passed the fall line and the onset of the instructions. Figure 5 (b) illustrates the average duration between turning points and the time when the system provided the instructions.

On average, the onset of tactile instructions coincided with the time when the snowboarders passed the fall line $(M(O)=$ $-.16, S D(O)=1.24$ second). Yet, the time offsets varied across the participants. The instructions were most delayed for participant $F$. He received instructions on average 0.56 second after passing turning points. This indicates that he skidded the snowboard. The system recognized the pivoting process when he exerted more pressure on the new riding edge after passing the fall line. The instructions were also delayed for participant $I$ who commented that he noticed the instruction at the shoulder toward the end of a turn. These delayed instructions could have interfered with the body movements that these snowboarders had to perform in order to return to neutral position. Despite the delay, both $F$ and $I$ considered the instructions to be helpful for riding switch.

Participant $J$ received instructions on average 1.61 second before passing turning points. This strongly suggests that he carved the turns at high speed, otherwise he could not have pivoted the snowboard as early before passing the fall line. In fact, $J$ was an advanced snowboarder who practiced carving in the afternoon. The questionnaire revealed that $J$ did not consider the instructions to be helpful.

\section{The Snowboarders' Skills in Riding Switch}

Table 2 shows whether or not the participants had tried to ride switch before the course. The table also shows the course instructor's rating of their initial and final riding skills. Six participants $(C, E, F, G, H, I)$ had poor riding skills at the beginning (rating $\leq 3$ ).

The snowboard instructor who reviewed the footage did not know that the snowboarders had participated in a course. Nei- 


\begin{tabular}{|c|c|c|c|c|c|c|c|c|c|c|c|}
\hline & Participant & $\mathrm{A}$ & $\mathrm{B}$ & $\mathrm{C}$ & $\mathrm{D}$ & $\mathrm{E}$ & $\mathrm{F}$ & G & $\mathrm{H}$ & I & $\mathrm{J}$ \\
\hline Tactile instructions & Time of day & $\mathrm{m}$ & $\mathrm{a}$ & $\mathrm{m}$ & $\mathrm{a}$ & $\mathrm{m}$ & $\mathrm{a}$ & $\mathrm{m}$ & $\mathrm{a}$ & $\mathrm{m}$ & $\mathrm{a}$ \\
\hline \multirow{2}{*}{ Descents in the morning } & Wrong upper body rotation & 42.9 & 46.7 & 23.8 & 12.5 & 15.4 & 35.7 & 14.8 & 38.7 & 65.8 & 45.2 \\
\hline & Wrong weight distribution & 28.6 & 0.0 & 52.4 & 25.0 & 26.9 & 28.6 & 18.5 & 29.0 & 42.1 & 22.6 \\
\hline \multirow{2}{*}{ Descents in the afternoon } & Wrong upper body rotation & 40.7 & 25.0 & 20.0 & 10.7 & 43.5 & 26.9 & 33.3 & 18.9 & 48.1 & 25.0 \\
\hline & Wrong weight distribution & 29.6 & 16.7 & 50.0 & 7.1 & 26.1 & 11.5 & 37.0 & 16.2 & 40.7 & 32.1 \\
\hline
\end{tabular}

Table 3. The instructions were provided during descents in the morning or in the afternoon (m/a). Percentage of riding mistakes when riding without tactile instructions (normal font) and with tactile instructions (bold font).

ther did he know that they had learned to ride switch with tactile instructions. He assessed the participants' skills based on the number of riding mistakes. Table 3 shows the average percentage of wrong upper body rotation and weight distribution for descents without and with tactile instructions.

The participants $A, C, E, G$ and $I$ received tactile instructions during lessons in the morning (Table 3). $E$ and $G$ most benefitted from these instructions. They descended more often with correct upper body posture $(E, G)$ and with correct weight distribution $(G)$ in the morning than when riding without tactile instructions in the afternoon. $A$ and $C$ had similar riding skills throughout the day. Participant $I$ improved his upper body posture in the afternoon.

The participants $B, D, F, H$ and $J$ received tactile instructions during lessons in the afternoon (Table 3). D, $F$ and $H$ made fewer mistakes during these rides, but their improved performance could also be attributed to the practice trials in the morning. $B$ and $J$ made fewer upper body mistakes, but they rode more often with wrong weight distribution in the afternoon than in the morning. The reason for these additional mistakes could be the more demanding carving technique that these snowboarders practiced in the afternoon.

For further data analysis we performed a repeated measures ANOVA with RidingCondition and Time as factors. Regarding the percentage of incorrect upper body rotation, there was no significant main effect of Time, no significant main effect of RidingCondition, and no significant interaction effect.

For weight distribution, there was no significant main effect of Time and no significant main effect of RidingCondition. There was a significant interaction effect of Time $x$ RidingCondition $\left(F_{1,8}=7.96, p=.023, r=.70\right)$. The post-hoc t-test showed some significant effects with large effect sizes: in the afternoon the traditional lessons lead to more mistakes ( $M=36.7, S E=4.22$ ) than riding with tactile instructions $(M=16.7, S E=4.22, t(14.46)=2.81, p=.014, r=$ $.59)$; lessons with tactile instructions lead to more mistakes in the morning $(M=33.7, S E=6.01)$ than in the afternoon $(M=16.7, S E=4.22, t(14.46)=2.39, p=.031, r=$ $.53)$; traditional lessons lead to fewer mistakes in the morning $(M=21.0, S E=5.39)$ than in the afternoon $(M=$ $36.7, S E=4.22, t(14.46)=-2.20, p=.044, r=.50)$.

Overall, it seems that traditional lessons were effective in the morning, whereas lessons with tactile instructions were effective in the afternoon. If we consider the participants' comments (see next section), one reason for these results could be that spoken instructions outweighed tactile instructions when practicing new exercises, whereas tactile instructions were considered to be helpful when advancing in the course.

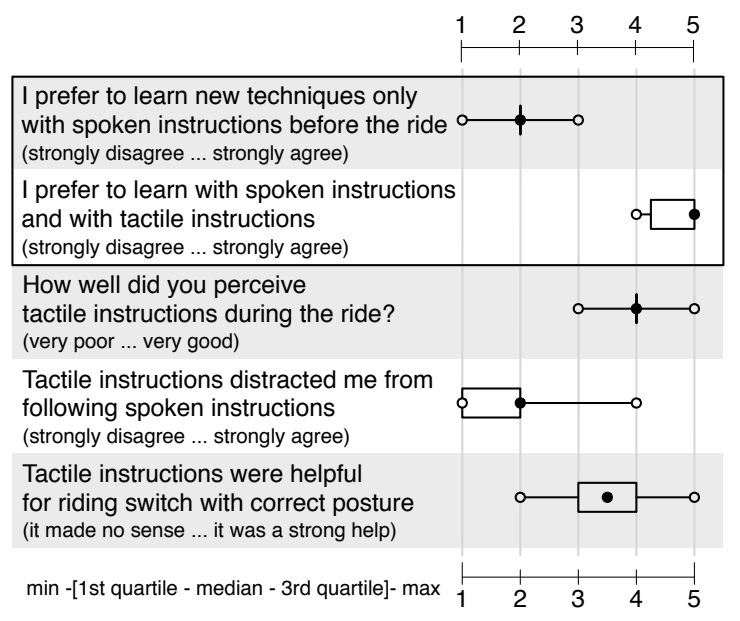

Figure 6. Likert-scale ratings of tactile instructions.

Although the main effect of RidingCondition was not significant, tactile instructions had a different effect on the type of riding mistakes, considering all descents and ignoring Time. For upper body rotation, there is a noticeable difference between riding with tactile instructions $(M=26.9, S E=$ $5.15)$ and traditional lessons $(M=36.4, S E=3.72)$. The post-hoc t-test shows a marginal significance with a large effect size $(t(8)=2.09, p=.070, r=.59)$. For weight distribution the difference between tactile $(M=25.2, S E=4.47)$ and traditional lessons $(M=28.9, S E=4.15)$ is small and has a small effect size $(t(8)=.89, p=.40, r=0.30)$.

This observation indicates that the instruction for upper body posture was more effective than the instruction for weight distribution. The reason for this deviation could be that maintaining proper weight distribution requires lots of practice to overcome the feeling of unease when leaning towards the fall line. Another reason could be that paying attention to two riding mistakes and to two tactile instructions is too demanding. It might be beneficial to address only one mistake at a time. In fact, participant $I$ commented that he could not attend to both tactile instructions. He focused on the instruction that was applied either to the shoulder or to the thigh.

\section{The Snowboarders' Opinion on Tactile Instructions}

The questionnaire revealed that all participants uniformly agreed to have much improved their skills in riding switch. Half of the group $(B, F, H, I, J)$ considered this riding technique to be difficult to learn.

Figure 6 shows the participants' rating of tactile instructions. Overall, they preferred lessons with tactile instructions over traditional lessons (Wilcoxon signed-ranks test for the framed 


\begin{tabular}{|lllllllllll|}
\hline Participant & A & B & C & D & E & F & G & H & I & J \\
\hline Instructions were helpful & y & $\mathbf{u}$ & $\mathbf{u}$ & $\mathbf{n}$ & y & y & y & u & y & $\mathbf{n}$ \\
T. i. corresponded to s. i. & y & s & y & s & y & y & y & s & y & s \\
\hline
\end{tabular}

Table 4. The participants' individual comments on tactile instructions (negative comments are shown in bold font): The instructions were helpful for riding switch (yes/no/undecided). The instructions corresponded to spoken instructions (yes/somewhat).

questions: $T=0, n=10, p<.01, r=-.64)$. Moreover, they reported that they did not have difficulty in perceiving the instructions. Nine participants agreed that the tactile instructions did not distract them from carrying out the spoken instructions, which the coach gave before the ride.

Eight participants explained why they valued tactile instructions: (A) "Direct feedback during the ride."; $(B)$ "Quite accurate."; $(C)$ "Clear enough to notice and locate, but not too strong to distract."; $(D, F, I)$ "The feedback at the thigh reminded me of correct position and kept me thinking about the weight distribution."; (I) "It was great fun and I would recommend it for snowboard students very much!"; $(E)$ "The feedback reminded me of correct basic posture when advancing in the course."; $(H)$ "The feedback was an additional clue to activate involved body parts."

Although all participants favored lessons with tactile instructions, they had different opinions whether or not these instructions were helpful for riding switch in our training scenario (Figure 6). Half of the group considered the instructions to be helpful (Table 4, row 1). The other participants were undecided $(B, C, H)$, or they stated that the instructions did not help $(D, J)$. We can clarify these different opinions if we consider the participants riding skills (Table 2), the exercises that they practiced, and their individual comments.

The following comments illustrate the participants' experiences with tactile instructions: $(B)$ "It was hard to process this information while trying to follow the spoken instructions."; $(C)$ "Since riding switch was completely new to me, I was very concentrated on not falling (in the beginning) and tried to follow the spoken instructions. I did not really react to the tactile instructions, although I noticed them, because there were so many other things to think about. I guess it would have been easier for me to regard them as additional instructions after some time of practice, once the basic way of riding was familiar to me, and when I could spend attention on more exact riding, e.g., at the end of the day."; (D) "I was so concentrating on the speed and movements and didn't actually keep my attention on the tactile feedback. I felt the feedback, but didn't actually follow it. I tried more to follow the instructions of the teacher."; $(H)$ "When trying to follow the spoken instructions, it was hard to pay active attention to the tactile feedback."; $(J)$ "I could not really pay attention to the tactile instructions because I was so concentrating on doing what the teacher told me to do."

The comments show that these participants focused on the coach's instructions, i.e., the spoken instructions outweighed the tactile instructions. This focus on spoken instructions is understandable because they practiced new exercises: $C$ and $H$ rode switch for the first time; $B, D$ and $J$ practiced carving. Moreover, these exercises caused higher cognitive load.
The participants had difficulty attending to tactile instructions while focusing on spoken instructions. In contrast, the participants who had some experience in riding switch and who did not practice carving did not mention having any difficulties paying attention to tactile instructions in addition to spoken instructions. In this case, the task was not too demanding.

These comments suggest that tactile instructions should be introduced later on during training. In fact, $J$ mentioned that tactile instructions could help snowboarders to correct and maintain proper posture at a later stage during training when they can ride better. $E$ shared his point of view: "When I was trying something new the extra feedback was a bit too much, but later, when repeating what I learned, it was very helpful." For this reason, we argue that $B, C, D, H$ and $J$ would have been able to attend to tactile instructions in a different training scenario, for example with less difficult exercises, or without receiving spoken instructions before descending the slope.

Another reason why $B, D$ and $J$ did not consider tactile instructions to be helpful could relate to carving. These participants practiced carving with tactile instructions that represented possible riding mistakes. They stated that the instructions somewhat corresponded to the coach's instructions (Table 4, row 2). Apparently, this use of tactile stimuli was not helpful in our training scenario.

We also found that the participants had a different opinion on how often the system should give instructions. Three inexperienced snowboarders $(E, H, I)$ preferred to receive tactile instructions for every turn. All other snowboarders stated that the system should only give instructions if they make a mistake. This dissent could indicate that the coach should also consider individual learning preferences for adjusting the amount of realtime instructions.

Some participants commented on the wearable system. Their main critique addressed the sensor cables inside the snowboard pants and the fact that the system triggered tactile instructions even while pausing on the slope. Ideally, a wearable training system should perform activity recognition in order to discriminate between riding and pausing [15].

\section{Study Limitations}

The results presented in this work have to be interpreted with caution because there are many variables that we could not control in our field study, for example, the participants' fatigue and motivation during the course, and the difficult exercises that the instructor issued to advanced snowboarders during lessons in the afternoon. Moreover, snowboarding is difficult to learn. A short-term study with few participants and with few descents can only provide initial insights into the use of tactile instructions in sports training.

There are several other issues that are difficult to deal with in field studies. For example, it is often difficult for coaches to assess a snowboarder's posture and performance from a distance. For this reason, our results are biased towards the viewpoint of the coach who conducted the course and the instructor who analyzed the footage. In addition, the snowboarders could have intentionally paid more attention to riding with correct posture because they were videoed. 
The technology also has to be considered. Our wearable system did not detect snowboarding mistakes. The participants received instructions for every turn, even if their posture was correct. The system also missed some turns. In this case, the snowboarders did not receive instructions. Furthermore, the participants' different riding skills and the exercises that they practiced influenced the way that the system responded to the movements of the snowboard. In particular, the time differed when the participants received instructions while pivoting.

The novelty of the technology is another aspect that could have influenced the participants' opinion. All snowboarders experienced for the first time tactile instructions. The benefits that these instructions could offer during training might not have been obvious to all of them. The experiences that users make with new technology can change over time, and the usefulness and long-term usability of the technology might become apparent only after prolonged use [11].

\section{IMPLICATIONS FOR SPORTS TRAINING}

The goal of this work was to identify conditions under which tactile instructions can support snowboarders in acquiring new skills, and conditions that should be avoided. In our training scenario, in addition to spoken instructions, amateur snowboarders received tactile instructions for learning a new riding technique. These tactile instructions were provided for every trial independent of whether the snowboarders made a mistake or not.

Our findings suggest that tactile instructions could potentially help snowboarders to reduce the number of riding mistakes and to improve their skills. Even so, our findings also revealed that it is important to consider the difficulty of the task that the learner performs, the learner's needs during training, and also the learner's preferences. Regarding our research questions, we can summarize our findings as follows:

Can snowboarders perceive tactile instructions when they practice a new riding technique in a realistic scenario? All snowboarders reported that they perceived the tactile instructions, even though their riding skills and the difficulty of the exercises differed. This result is surprising because it contrasts with the findings of other studies where the participants could not reliably locate [2] or could not perceive [21] tactile stimuli in situations where they experienced high cognitive load. We surmise that this could relate to the characteristics of our stimuli. In contrast to [2, 21], which used localized pulses, our stimuli were based on directional lines that stimulated a larger area of the skin. These spatiotemporal patterns could have promoted the perception of the tactile stimuli. Future studies should investigate if such patterns are indeed beneficial in situations of higher cognitive load.

Can they attend to these instructions? The snowboarders who did not have previous experience in riding switch and the snowboarders who practiced new exercises focused on the coach's instructions. This focus on spoken instructions, and the cognitive load participants experienced because they practiced new exercises, made it difficult to pay attention to tactile instructions during the ride. Unlike these participants, the snowboarders who already had some experience in riding switch were able to attend to tactile instructions in addition to spoken instructions. Overall, this finding corresponds to findings of other studies that investigated tactile instructions for teaching motor skills. For example, van der Linden et al. [21] pointed out that the attentional demands of the performed task can influence if the learner can attend to vibrotactile feedback. In our case, however, the results also indicate that some participants received too many instructions. The combination of spoken instructions and tactile instructions made it difficult for some participants to also attend to tactile instructions when they experienced high cognitive load, although perceiving the tactile instructions was not problematic. Thus, besides finding stimuli that learners can perceive and attend to under high cognitive load, it is also important to find the right balance between spoken instructions and tactile instructions.

How helpful do they consider these instructions? All snowboarders stated to prefer lessons with tactile instructions to traditional lessons where the coach gave only spoken instructions before the ride and feedback after the ride. This strongly suggests that they considered tactile instructions to be potentially useful during training. Even so, only the snowboarders who practiced exercises that were not too difficult benefitted from tactile instructions and considered these instructions to be helpful for learning to snowboard.

Furthermore, some advanced snowboarders practiced exercises where tactile instructions signaled possible riding mistakes. Apparently, these instructions were not helpful in our training scenario. This could indicate that tactile stimuli should not signal riding mistakes. A better approach could be to guide the snowboarder how to move correctly during the next trial. This is what coaches do. They do not say that movements are wrong. They say how to improve the posture.

Based on our findings, we conclude that tactile instructions should not be applied during the first lessons or when the coach introduces new exercises. In these cases, spoken instructions before the ride could outweigh tactile instructions even if tactile instructions are provided concurrently during the execution of the movements. Moreover, the learner will experience higher cognitive load when practicing new body movements and might not be able to attend to tactile instructions in addition to spoken instructions. Therefore, tactile instructions should be introduced after the learner has acquired the basic skills in performing the new task. At this stage during training, the cognitive load on the learner will be lower. Tactile instructions could support the learner in practicing and fine-tuning the previously learned movements.

To conclude, we propose a training scenario where tactile instructions will be gradually introduced: The coach gives spoken instructions for a new exercise as usual. After the learner acquires the basic skills for performing the exercise, the coach will introduce one tactile instruction to correct the body movement that he considers most important for improving the learner's skills. The wearable training system will provide this instruction frequently for every trial in order to allow the learner to focus on this particular movement. The frequency of this instruction will be reduced when the learner becomes more proficient in performing the exercise, and will 
be eventually omitted when the performance becomes stable. If the learner makes the mistake again later on, however, the tactile instruction will be reintroduced as a reminder.

A coach who observes a particular riding mistake could manually trigger a tactile instruction in the aforementioned scenario. Even so, we surmise that this instruction could be delayed. As the timing of the instruction is important, a sensing system may be more precise in providing realtime instructions, in particular if the system can accurately classify a specific body movement or riding mistake. Besides assisting snowboarders during courses and when riding alone, coaches could also benefit from such a system because they cannot always observe and correct every student separately. Moreover, coaches could miss some incorrect body movements when observing students from a distance.

\section{CONCLUSIONS}

This work explored the use of realtime tactile instructions for teaching sport skills in a realistic scenario. We conducted a snowboarding course with amateur snowboarders who received tactile instructions while descending the slope. These instructions signaled which body movements to perform. A wearable system automatically provided these instructions for every trial, in addition to the spoken instructions and the feedback that the coach gave before and after the ride. The tactile instructions were most effective when the exercises that the snowboarders practiced were not too difficult. When the snowboarders practiced new and difficult exercises, however, they focused on the coach's instructions and had difficulty paying attention to tactile instructions. Overall, our findings indicate that tactile instructions could support snowboarders in improving their performance. Moreover, our participants considered these instructions to be potentially useful during training. Based on our findings, we can conclude that tactile instructions are a viable approach for supporting learners in acquiring sport skills if these instructions are tailored to meet the learners' needs during training.

As future work, we would like to validate our findings in other training settings and for other physical activities. We intend to conduct a long-term study where tactile instructions are gradually introduced during training. Furthermore, we plan to investigate realtime spoken instructions that are provided over earplugs. Finally, we would like to develop a wearable system that can recognize snowboarding mistakes and that can support snowboarders in learning different riding techniques.

\section{REFERENCES}

1. Baca, A., Kornfeind, P. Rapid Feedback Systems for Elite Sports Training. IEEE Pervasive Computing 5, 4, (2006), 70-76.

2. Bhargava, A., Scott, M., Traylor, R., Chung, R., Mrozek, K., Wolter, J., Tan, H. Z. Effect of Cognitive Load on Tactor Location Identification in Zero-g. Proc. WHC 2005, IEEE Computer Society, 56-62.

3. Bloomfield, A., Badler, N. I. Virtual Training via Vibrotactile Arrays. Presence: Teleoperators and Virtual Environments 17, 2, (2008), 103-120.

4. Chi, E. H., Borriello, G., Hunt, G., Davies, N. Guest Editors' Introduction: Pervasive Computing in Sports
Technologies. IEEE Pervasive Computing 4, 3, (2005), 22-25.

5. Dt. Verband f. d. Skilehrwesen, Interski Deutschland. Snowboard-Lehrplan. BLV Buchverlag, München. 2003.

6. Feygin, D., Keehner, M., Tendick, F. Haptic Guidance: Experimental Evaluation of a Haptic Training Method for a Perceptual Motor Skill. Proc. HAPTICS 2002, IEEE Computer Society, 40-47.

7. Geldard, F. A. Sensory Saltation: Metastability in the Perceptual World. Lawrence Erlbaum Associates. 1975.

8. Hoggan, E., Crossan, A, Brewster, S. A., Kaaresoja, T. Audio or Tactile Feedback: Which Modality When? Proc. CHI 2009, ACM Press, 2253-2256.

9. Holland, S., Bouwer, A. J., Dalgelish, M., Hurtig, T. M. Feeling the Beat Where it Counts: Fostering Multi-Limb Rhythm Skills with the Haptic Drum Kit. Proc. TEI 2010, ACM Press, 21-28.

10. Huang, K., Starner, T., Do, E., Weiberg, G., Kohlsdorf, D., Ahlrichs, C., Leibrandt, R. Mobile Music Touch: Mobile Tactile Stimulation for Passive Learning. Proc. CHI 2010, ACM Press, 791-800.

11. Karapanos, E., Zimmerman, J., Forlizzi, J., Martens, J.B. User Experience Over Time: An Initial Framework. Proc. CHI 2009, ACM Press, 729-738.

12. Kwon, D. Y., Gross, M. Combining Body Sensors and Visual Sensors for Motion Tracking. Proc. Advances in Computer Entertainment Technology, 2005 ACM Press, 94-101.

13. Lieberman, J., Breazeal, C. TIKL: Development of a Wearable Vibrotactile Feedback Suit for Improved Human Motor Learning. IEEE Transactions on Robotics, (2007), 919-926.

14. Nakamura, A., Tabata, S. Ueda, T. Kiyofuji, S., Kuno, Y. Multimodal Presentation Method for a Dance Training System. Proc. CHI 2005 Extended Abstracts, ACM Press, 1685-1688.

15. Spelmezan, D., Schanowski, A., Borchers, J. Wearable Automatic Feedback Devices for Physical Activities. Proc. BodyNets 2009, ICST, 1-8.

16. Spelmezan, D., Jacobs, M., Hilgers, A., Borchers, J. Tactile Motion Instructions for Physical Activities. Proc. CHI 2009, ACM Press, 2243-2252.

17. Tan, H., Lim, A., Traylor, R. A Psychophysical Study of Sensory Saltation with an Open Response Paradigm. Proc. ASME 2000, 1109-1115.

18. Takahata, M., Shiraki, K., Sakane, Y., Takebayashi, Y. Sound Feedback for Powerful Karate Training. Proc. NIME 2004, National University of Singapore, 13-18.

19. Athletic Harmony. News Track in Communications of the ACM 51, 6, (2008), 7.

20. van Erp. J. B. F., Saturday, I., Jansen, C. Application of tactile displays in sports: where to, how and when to move. Proc. EuroHaptics 2006, 90-95.

21. van der Linden, J., Johnson, R., Bird, J., Rogers, Y., Schoonderwaldt, E. Buzzing to Play: Lessons Learned From an In the Wild Study of Real-time Vibrotactile Feedback. Proc. CHI 2011, ACM Press, 533-542.

22. Wulf, G. Attention and Motor Skill Learning. Human Kinetics. 2007. 